EPJ Web of Conferences 60, 19003 (2013)

DOI: $10.1051 /$ epjconf $/ 20136019003$

(C) Owned by the authors, published by EDP Sciences, 2013

\title{
Measurement of diboson production with the ATLAS detector
}

\author{
Suen $\mathrm{Hou}^{1, \mathrm{a}}$, on behalf of the ATLAS collaboration \\ ${ }^{1}$ Academia Sinica, Taipei
}

\begin{abstract}
ATLAS measurements of diboson production processes involving combinations of $W, Z$ and isolated photons are summarized. Measurements using data at $7 \mathrm{TeV}$ as well as more recent results using data at $8 \mathrm{TeV}$ are presented. The measurements are performed using leptonic decay modes, including the invisible decay $Z \rightarrow v \bar{v}$, as well as semileptonic channels. Differential and total cross sections are presented and are used to place constraints on anomalous triple-gauge boson couplings. An overview of these results is given.
\end{abstract}

\section{Introduction}

The large data statistics collected by ATLAS [1] in 2011 and 2012, in LHC $p p$ collisions at $\sqrt{s}=7 \mathrm{TeV}$ and $8 \mathrm{TeV}$, respectively, provide precision measurements of the diboson production mechanism described by the Electroweak sector of the Standard Model (SM). All analyses make use of leptonic decay of $W$ and $Z$ bosons, for diboson processes of $W W, W Z, Z Z, W \gamma$ and $Z \gamma$. The integrated luminosities of analyzed data corresponds to $4.6 \mathrm{fb}^{-1}$ at $\sqrt{s}=7 \mathrm{TeV}$ and up to $20 \mathrm{fb}^{-1}$ at $\sqrt{s}=8 \mathrm{TeV}$. The production cross sections are known to next-to-leading order (NLO), and the electroweak radiation corrections are important at high $\sqrt{s}[2,3]$. Therefore the cross-section measurements provide sensitive tests of the electroweak interaction at the $\mathrm{TeV}$ scale.

Diboson channels are also sensitive to new physics phenomena, either through resonant production of new particles or through anomalous contribution to Triple Gauge couplings (TGCs). Interactions between gauge boson are predicted by the SM. Any deviation of the triple gauge couplings from the SM prediction will enhance diboson production with higher invariant masses. Studies of diboson events are candles to improve understanding of background processes in searches of SM Higgs boson and new physics at the high energy frontier.

In the following we present measurements of diboson production cross sections and searches of anomalous contributions to SM predicted triple gauge couplings.

\section{Cross-section measurements}

All the analyses requires leptons (electrons or muons) originated from the decay of a $W$ or $Z$ boson with large transverse momentum within the detector fiducial region of pseudo-rapidity $|\eta|<2.47$ for electrons (excluding the barrel-endcap transition region of $1.36<|\eta|<1.52$ ) and

\footnotetext{
a e-mail: suen.hou@cern.ch
}

$|\eta|<2.4$ for muons. The selection criteria vary slightly on $|\eta|$ ranges and $p_{\mathrm{T}}$ thresholds for leptons in different diboson channels. To reject leptons from fragmentation of jets, isolation cuts are imposed. Jets are built by clustering energy depositions in the calorimeter using the anti-kT algorithm with radius parameter 0.4 . The neutrino from $W$ decay is inferred by the missing transverse energy $E_{\mathrm{T}}^{\mathrm{miss}}$ calculated using reconstructed physics objects and calorimeter energy depositions not associated to physics objects.

The measurements of diboson production cross sections in the detector fiducial region are derived with the observed events and corrections for the event selection and reconstruction efficiencies. The total cross sections of $p p \rightarrow V V$ (with $V=W, Z$ ) are derived with extrapolation to the full phase space and branching ratio of $W$ and $Z$ decay channels of the event selection. For the $V \gamma$ channels, only fiducial cross sections are measured to avoid collinear and infrared divergences. The event selection criteria of each diboson processes are described in the following. The measurements are compared to the next-toleading order calculations of various event generators including MCFM [4] and using the CT10 [5] and MSTW [6] parton distribution functions.

\section{$2.1 W^{+} W^{-} \rightarrow \ell^{+} v \ell^{\prime-} v^{\prime}$ channels}

The $W^{+} W^{-}$production cross section is measured using the full $7 \mathrm{TeV}$ dataset of $4.6 \mathrm{fb}^{-1}$ [7]. Only leptonic decays of both $\mathrm{W}$ bosons are used, leading to a final state of two leptons and large event $E_{\mathrm{T}}^{\text {miss }}$. Backgrounds consist of $t \bar{t}$, $W / Z+$ jets and other diboson processes. To distinguish the signal from the large $W / Z+$ jets and $t \bar{t}$ backgrounds, events are required to have large missing transverse energy and no high transverse momentum jets. Resonant $Z$ events are removed by a mass window cut of $\left|m_{\ell \ell^{\prime}}-m_{Z}\right|>15 \mathrm{GeV}$.

The observed distribution of the transverse mass calculated from the two leptons and $E_{\mathrm{T}}^{\mathrm{miss}}$ is shown in Figure 1. The measured cross sections [7] are listed in Table 1, and they agree within errors with the SM NLO predictions. 

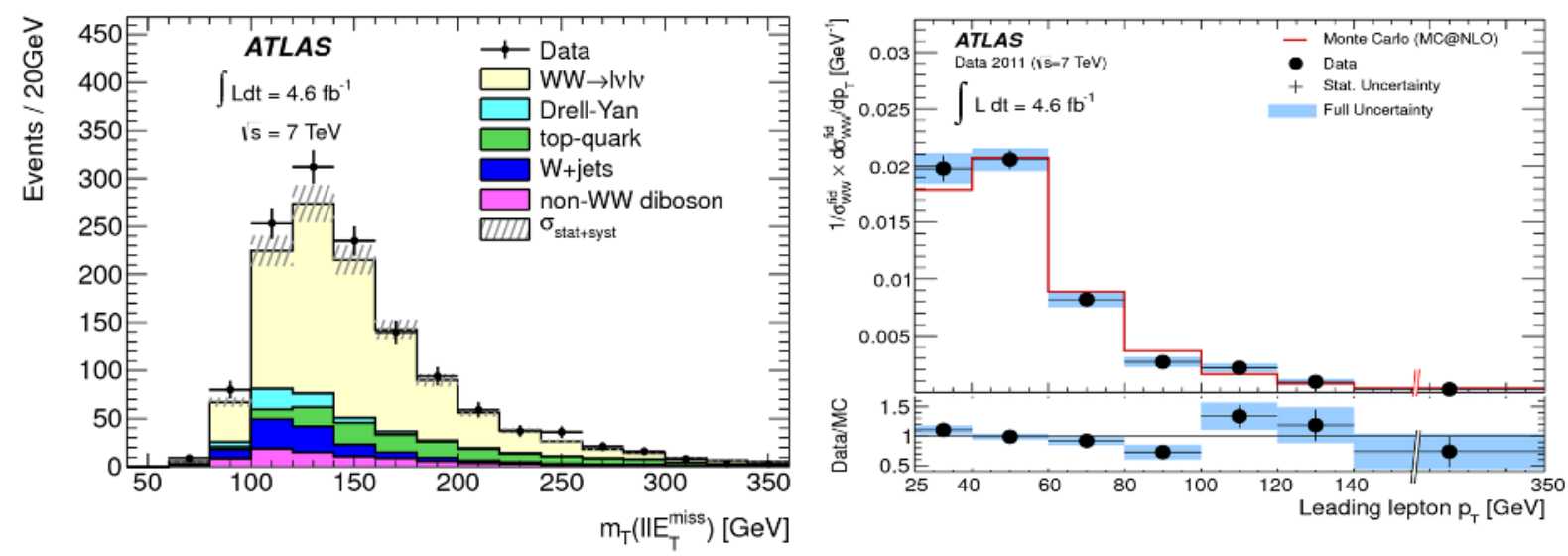

Figure 1. Distribution of transverse mass of the $\ell \ell^{\prime}+E_{\mathrm{T}}^{\text {miss }}$ (left) for selected $W W$ events, and the normalized differential cross section in detector fiducial region as a function of the leading lepton $p_{\mathrm{T}}[7]$.
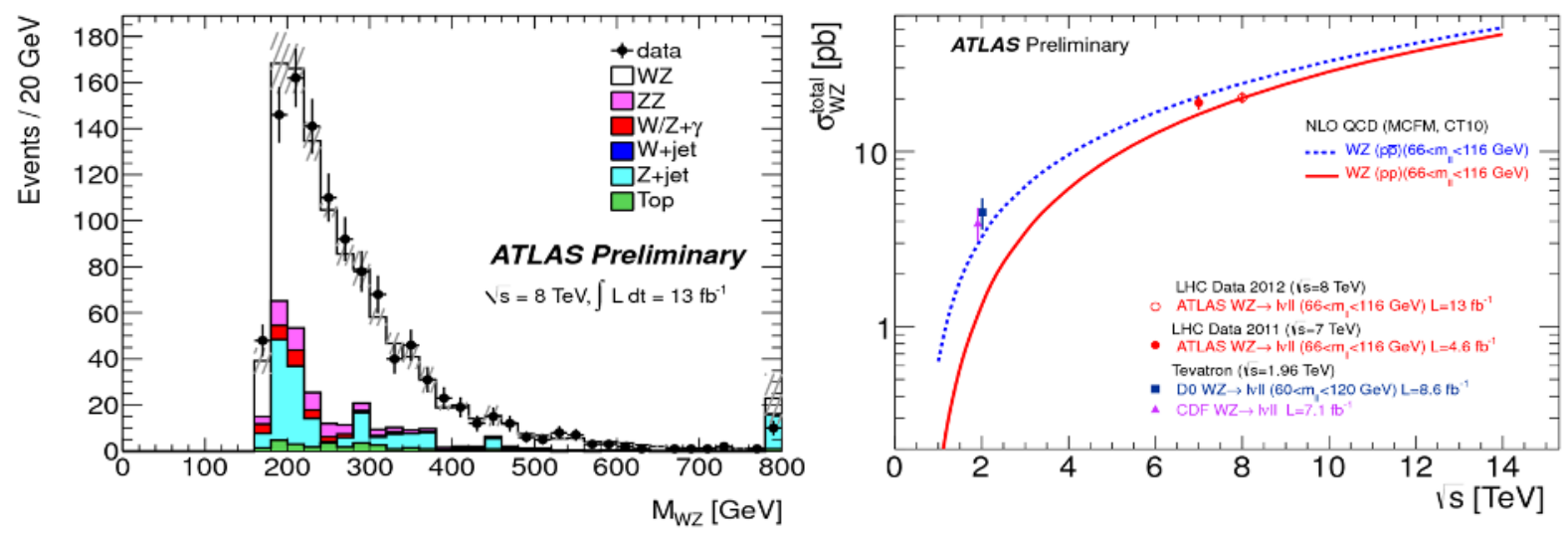

Figure 2. Distribution of the $W Z$ invariant mass (left), using $13 \mathrm{fb}^{-1}$ of $\sqrt{s}=8 \mathrm{TeV}$ data. Measurements and theoretical predictions are plotted (right) for the total WZ production cross section as a function of center-of-mass energy [9].

The normalized differential cross section as a function of the leading lepton $p_{\mathrm{T}}$ is illustrated in Figure 1.

\section{$2.2 W^{ \pm} Z \rightarrow \ell^{ \pm} v \ell^{\prime+} \ell^{\prime-}$ channels}

$W^{ \pm} Z$ events using leptonic decays are analyzed from the $7 \mathrm{TeV}$ data $\left(4.6 \mathrm{fb}^{-1}\right)[8]$, and the first part of the $8 \mathrm{TeV}$ data corresponding to $13 \mathrm{fb}^{-1}$ [9]. The events are required to have three isolated leptons with large $E_{\mathrm{T}}^{\text {miss }}$. Two leptons are required to form the $Z$ boson with the invariant mass within $10 \mathrm{GeV}$ of the $Z$ mass. The background events include $Z+$ jets and $t \bar{t}$. The systematic uncertainties mainly come from lepton and $E_{\mathrm{T}}^{\text {miss }}$ reconstructions.

The observed $W^{ \pm} Z$ transverse mass distribution of the $8 \mathrm{TeV}$ data is shown in Figure 2. The measured cross sections are listed in Table 1. Comparison with the theoretical calculations are illustrated in Figure 2 for both 7 and $8 \mathrm{TeV}$ data.

\subsection{Semileptonic $W W, W Z \rightarrow \ell v j j$ channels}

The measurements of semileptonic diboson production is conducted with the $7 \mathrm{TeV}$ data $\left(4.6 \mathrm{fb}^{-1}\right)[10]$ for $W W$ and
$W Z$ channels requiring one high- $p_{\mathrm{T}}$ lepton of $W$ decay and two jets from a boson ( $W$ or $Z$ ) decay. Background is dominated by the irreducible $W+$ jets events. The combined cross section of $W W$ and $W Z$ is listed in Table 1 . The large systematic uncertainty is mainly due to uncertainties in the background estimation.

\section{$2.4 Z Z \rightarrow \ell^{+} \ell^{-} \ell^{\prime+} \ell^{\prime-}, \ell^{+} \ell^{-} v \bar{v}$ channels}

The $Z Z$ production cross section is studied from $7 \mathrm{TeV}$ data $\left(4.6 \mathrm{fb}^{-1}\right)$ [11] and the full $8 \mathrm{TeV}$ data $\left(20 \mathrm{fb}^{-1}\right)$ [12]. Events are selected in two decay modes of $Z Z \rightarrow$ $\ell^{+} \ell^{-} \ell^{\prime+} \ell^{\prime-}$ and $Z Z \rightarrow \ell^{+} \ell^{-} v \bar{v}$ (using $7 \mathrm{TeV}$ data).

The event selection for $Z Z \rightarrow 4 \ell$ requires exactly four high- $p_{\mathrm{T}}$ leptons, forming two opposite-sign same-flavor pairs. Ambiguities in pairing are resolved by minimizing the mass differences of the pairs to $Z$ mass. After requiring both $Z$ to be on-shell, with $Z$ pair mass within $66<m_{\ell^{+} \ell^{-}}<116 \mathrm{GeV}$, the candidate sample is very clean with background contribution of a few percents. Plotted in Figure 3 is the four-lepton invariant mass distribution obtained with the $8 \mathrm{TeV}$ data. 

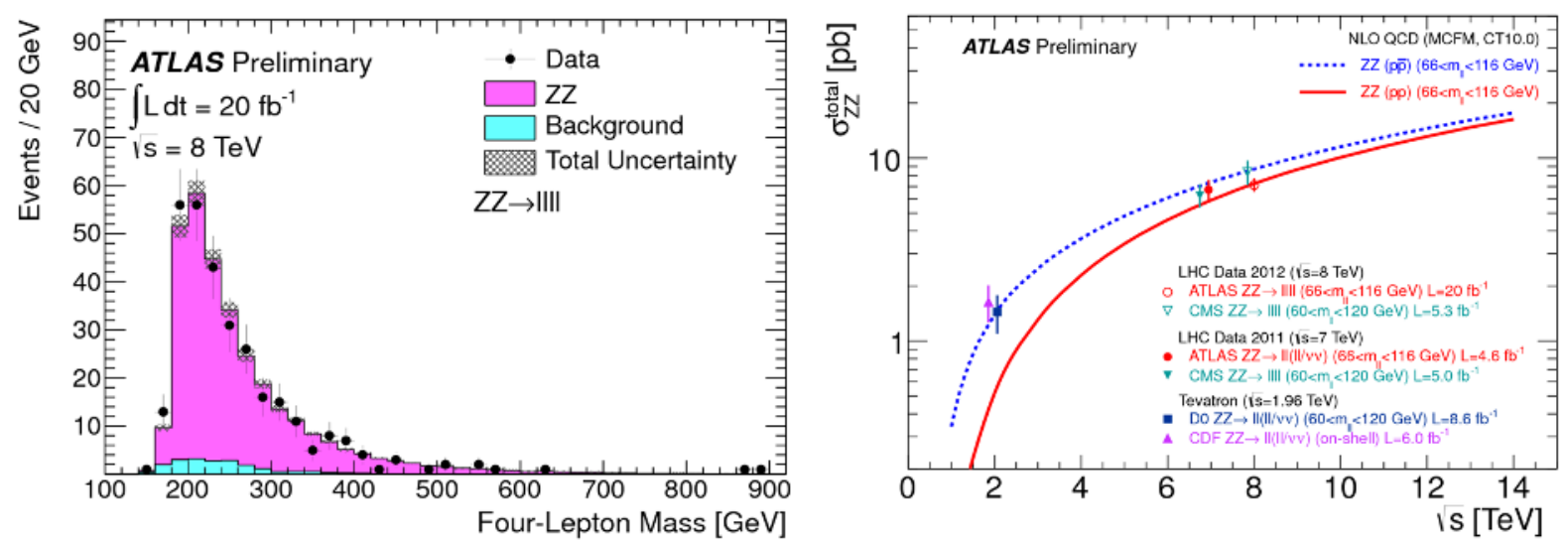

Figure 3. Invariant mass distribution for $Z Z$ candidates (left) in leptonic decay channels using full $8 \mathrm{TeV}$ data of 2012. Measurements and theoretical predictions are plotted (right) for the total ZZ production cross section as a function of center-of-mass energy [12].
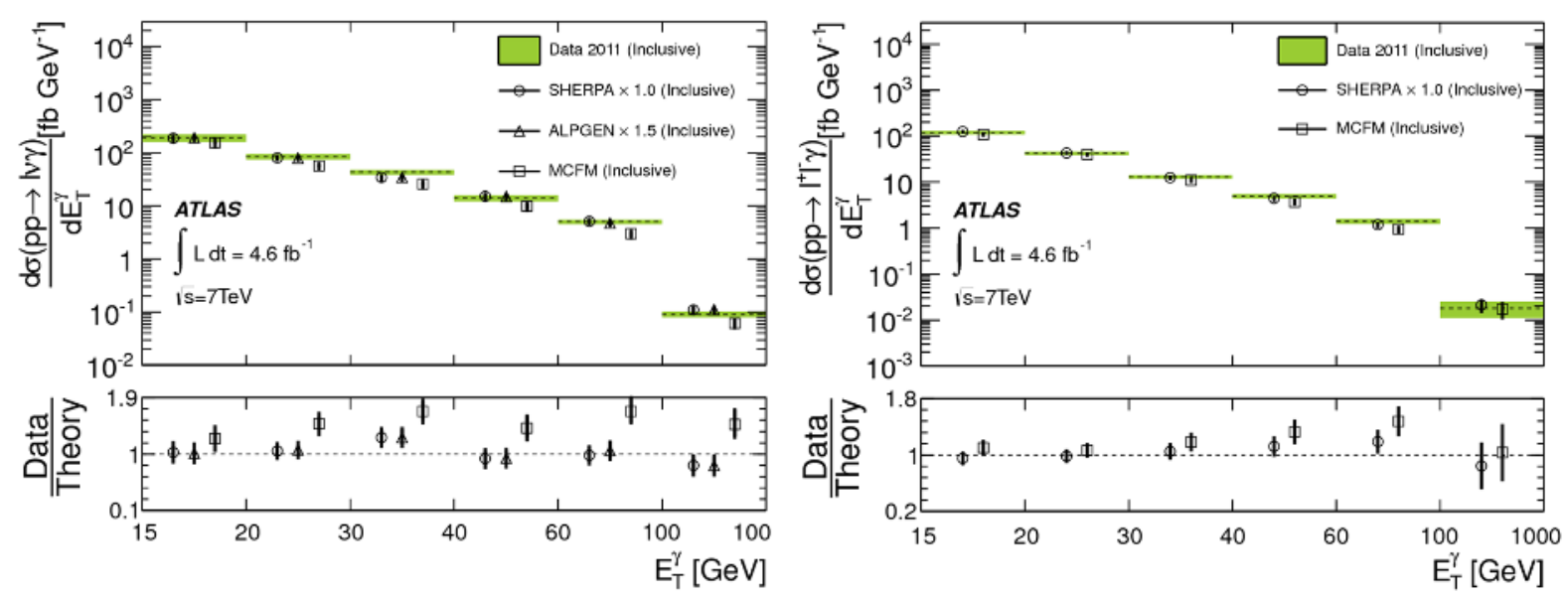

Figure 4. Differential cross sections of photon $E_{\mathrm{T}}$ of $W \gamma$ (left) and $Z \gamma$ (right) events. The lower plots show the ratio of the data to the predictions by different generators [13]).

The event sample for $\ell^{+} \ell^{-} v \bar{v}$ mode is selected from $7 \mathrm{TeV}$ data, with two opposite-sign same-flavor leptons and large event $E_{\mathrm{T}}^{\mathrm{miss}}$. The background includes $Z+$ jets, $t \bar{t}$ and other diboson processes. A mass window cut is required for the lepton pair. Events are further required to be without high- $p_{\mathrm{T}}$ jets to suppress $Z+$ jets and $t \bar{t}$ background. The projection of $E_{\mathrm{T}}^{\mathrm{miss}}$ along $p_{\mathrm{T}}^{Z}$ is required to exceed 75 $\mathrm{GeV}$.

The measured cross sections are listed in Table 1 , for $4 \ell$ mode of the $8 \mathrm{TeV}$ data and the combined $4 \ell$ and $\ell \ell v \bar{v}$ modes of the $7 \mathrm{TeV}$ data. Illustrated in Figure 3 is the comparison of cross-section measurements with SM NLO calculations for both 7 and $8 \mathrm{TeV}$ data. The measured cross sections are consistent within errors with the SM NLO predictions.

\section{$2.5 W^{ \pm} \gamma \rightarrow \ell^{ \pm} v \gamma, Z \gamma \rightarrow \ell^{+} \ell^{-} \gamma, v \bar{v} \gamma$ channels}

The $W^{ \pm} \gamma$ and $Z \gamma$ cross sections were measured using the $7 \mathrm{TeV}$ dataset corresponding to $4.6 \mathrm{fb}^{-1}$ [13]. The lower energy threshold for $W \gamma$ and $Z \gamma$ implies large production cross section. Photons can be radiated from the collid- ing patrons or from the $W$ boson. Additional contributions come from final state radiation (FSR) with the photon irradiated from decay leptons in $W$ and $Z$ events. The interference between these processes and the NLO corrections introduce large correction factor to high- $p_{\mathrm{T}}$ photons. Only leptonic decays of $\mathrm{W}$ and $\mathrm{Z}$, including $Z \rightarrow v \bar{v}$ are used. To suppress FSR events, photons are required to be separated from leptons $(\Delta R(\ell, \gamma)>0.7)$. Background includes events with jet-induced photons or jet-faking leptons. Major background processes are $W+$ jets and $\gamma+$ jets for $W \gamma$, $Z+$ jets for $Z(\rightarrow \ell \ell) \gamma$, and $Z+$ jets, $\gamma+$ jets and multijets for $Z(\rightarrow v \bar{v}) \gamma$.

Events are selected in the detector fiducial phase space listed in Table 1. The $W \gamma$ sample are required to have a lepton, a photon and large $E_{\mathrm{T}}^{\mathrm{miss}}$. The selection for $Z(\rightarrow$ $\ell \ell) \gamma$ requires a mass threshold of $m_{\ell^{+} \ell^{-}}>40 \mathrm{GeV}$. To suppress jet-induced background, $Z(\rightarrow v \bar{v}) \gamma$ events must have a very energetic photon of $E_{\mathrm{T}}>100 \mathrm{GeV}$ and $E_{\mathrm{T}}^{\text {miss }}>90$ $\mathrm{GeV}$.

Illustrated in Figure 4 are the differential cross sections of $W(\rightarrow \ell v) \gamma$ and $Z(\rightarrow \ell \ell) \gamma$ as functions of the photon $E_{\mathrm{T}}$, in comparison with the theoretical calculations. The cross- 
Table 1. Measured diboson production cross sections in comparison with the SM NLO calculations. Cross sections of massive diboson pairs ( $W W, W Z, Z Z$ ) are corrected to the full phase space. Measurements for $W \gamma$ and $Z \gamma$ are in fiducial regions as indicated.

\begin{tabular}{|c|c|c|c|c|}
\hline channel & $\begin{array}{l}\sqrt{s} \\
(\mathrm{TeV})\end{array}$ & $\begin{array}{l}\int L \\
\left(\mathrm{fb}^{-1}\right)\end{array}$ & $\begin{array}{l}\sigma(p p \rightarrow V V) \\
(\mathrm{pb})\end{array}$ & $\begin{array}{l}\text { SM NLO prediction } \\
(\mathrm{pb})\end{array}$ \\
\hline$W W \rightarrow \ell v \ell^{\prime} v^{\prime}$ & 7 & 4.6 & $51.9 \pm 2.0$ (stats.) \pm 3.9 (syst.) \pm 2.0 (lumi.) [7] & $44.7_{-1.9}^{+2.1}$ \\
\hline$W Z \rightarrow \ell v \ell^{\prime} \ell^{\prime}$ & 7 & 4.6 & $19.0_{-1.3}^{+1.4}$ (stat.) \pm 0.9 (syst.) \pm 0.4 (lumi.) $[8]$ & $17.6_{-1.0}^{+1.1}$ \\
\hline & 8 & 13 & $20.3_{-0.7}^{+0.8}$ (stat.) ${ }_{-1.1}^{+1.2}$ (syst.) ${ }_{-0.6}^{+0.7}$ (lumi.) [9] & $20.3 \pm 0.8$ \\
\hline$W W, W Z \rightarrow \ell v j j$ & 7 & 4.6 & $72 \pm 9$ (stat.) \pm 15 (syst.) \pm 13 (MC stats.) [10] & $63.4 \pm 2.6$ \\
\hline$Z Z \rightarrow \ell \ell \ell^{\prime} \ell^{\prime}, \ell \ell v \bar{v}$ & 7 & 4.6 & $6.7 \pm 0.7$ (stat.) ${ }_{-0.3}^{+0.4}$ (syst.) \pm 0.3 (lumi.) [11] & $5.89_{-0.18}^{+0.22}$ \\
\hline$Z Z \rightarrow \ell \ell \ell^{\prime} \ell^{\prime}$ & 8 & 20 & $7.1_{-0.4}^{+0.5}$ (stat.) \pm 0.3 (syst.) \pm 0.2 (lumi.) [12] & $7.2_{-0.2}^{+0.3}$ \\
\hline$W \gamma \rightarrow \ell v \gamma$ & 7 & 4.6 & \multicolumn{2}{|c|}{$p_{\mathrm{T}}^{\ell}>25 \mathrm{GeV}|\eta|<2.47 ; E_{\mathrm{T}}^{\gamma}>15 \mathrm{GeV},|\eta|<2.37 ; p_{\mathrm{T}}^{v}>35 \mathrm{GeV}$} \\
\hline$Z \gamma \rightarrow \ell \ell \gamma$ & 7 & 4.6 & \multicolumn{2}{|c|}{$p_{\mathrm{T}}^{\ell}>25 \mathrm{GeV},|\eta|<2.47 ; E_{\mathrm{T}}^{\gamma}>15 \mathrm{GeV},|\eta|<2.37 ; m(\ell \ell)>40 \mathrm{GeV}$} \\
\hline$Z \gamma \rightarrow v \bar{v} \gamma$ & 7 & 4.6 & $\begin{array}{l}p_{\mathrm{T}}^{\bar{v}}>90 \mathrm{GeV} ; E_{\mathrm{T}}^{\gamma}>100 \mathrm{GeV} \\
0.133 \pm 0.013 \text { (stat.) } \pm 0.020 \text { (syst.) } \pm 0.005 \text { (lumi.) [13] }\end{array}$ & $0.156 \pm 0.012$ \\
\hline
\end{tabular}

section measurements obtained in the fiducial regions are listed in Table 1.

\section{Anomalous Triple Gauge Coupling}

Contributions of anomalous triple gauge couplings (aTGCs) can be parametrized by effective Lagrangian with the couplings listed in Table $2[14,15,16]$. In the SM, charged TGCs contribute to $W W, W Z$ and $W \gamma$ channels, with the values of $\lambda_{V}=0$ and $g_{1}^{V}$ and $\kappa^{V}$ equal 1. Coupling between neutral bosons $(\mathrm{Z}, \gamma)$ are not present, namely $h_{i}^{Z, \gamma}=f_{i}^{Z, \gamma}=0$. The aTGCs are the variations of the TGCs from their SM values.

The anomalous couplings are assumed to have energy scale dependence. To avoid unitarity violation, the strength must vanish at high diboson invariant mass $(\sqrt{\hat{s}})$. A form-factor parametrization is applied to all the aTGCs with $\alpha(\hat{s})=\alpha_{0} /\left(1+\hat{s} / \Lambda^{2}\right)^{n}$, where $\Lambda$ is the cut-off scale with values chosen ( 2 or $3 \mathrm{TeV})$, and $n=2,3$, or $4 \mathrm{de}$ pending on the coupling.

Table 2. TGC vertices, and the corresponding diboson channels and couplings in the effective Lagrangians.

\begin{tabular}{ccc}
\hline vertex & channels & couplings \\
\hline$W W \gamma$ & $W W, W \gamma$ & $\lambda_{\gamma}, \Delta \kappa_{\gamma}$ \\
$W W Z$ & $W W, W Z$ & $\lambda_{Z}, \Delta \kappa_{Z}, \Delta g_{1}^{Z}$ \\
$Z Z \gamma$ & $Z \gamma$ & $h_{3}^{Z}, h_{4}^{Z}$ \\
$Z \gamma \gamma$ & $Z \gamma$ & $h_{3}^{\gamma}, h_{4}^{\gamma}$ \\
$Z \gamma Z$ & $Z Z$ & $f_{4}^{\gamma}, f_{5}^{\gamma}$ \\
$Z Z Z$ & $Z Z$ & $f_{4}^{Z}, f_{5}^{Z}$ \\
\hline
\end{tabular}

Non-zero aTGCs are predicted to contribute excess event rates in higher invariant mass region, and thus more high- $p_{\mathrm{T}}$ decay secondaries than the SM predictions. The observables to be examined are the kinematic distributions of $p_{\mathrm{T}}$ of bosons or their decay leptons. The likelihood function calculated by comparing the $p_{\mathrm{T}}$ spectrum to the predictions with varying aTGCs is used to set $95 \%$ confidence interval on the aTGCs.

Illustrated in figure 5 are the $95 \%$ C.L. limits of charged aTGCs for $W W Z$ and $W W \gamma$ vertices. Results are obtained from the $W W, W Z$ and $W \gamma$ analyses using $7 \mathrm{TeV}$ data $[7,8,13]$.

$W^{+} W^{-}$production is sensitive to aTGCs in the $W W \gamma$ and $W W Z$ vertices, and thus depends on all charged aTGCs. The $p_{\mathrm{T}}$ distribution of the leading lepton is used to derive aTGC limits for the "LEP scenario" [17] and the number of independent aTGCs is only three $\left(\Delta \kappa_{Z}, \lambda_{Z}\right.$ and $\left.\Delta g_{1}^{Z}\right) . \quad W^{ \pm} Z$ and $W \gamma$ events are sensitive to $W W Z$ and $W W \gamma$ vertices, respectively. The $p_{\mathrm{T}}(Z)$ spectrum of $W Z$ sample is used to derive aTGCs of $W W Z$, and the photon spectrum of $W \gamma$ sample is used for the two aTGCs of the $W W \gamma$ vertex.

The effective Lagrangians for $Z Z$ and $Z \gamma$ channels differ for the different vertices. The $Z Z$ production is sensitive to the $Z Z Z$ and $Z \gamma Z$ vertices. Using the $7 \mathrm{TeV}$ data, $95 \%$ confidence intervals are set for the $f_{4}^{\gamma}, f_{5}^{\gamma} f_{4}^{Z}$, and $f_{5}^{Z}$ and shown in Figure 6 [11].

The photon spectrum, particularly at high photon energies, is sensitive to the $Z Z \gamma$ and and $Z \gamma \gamma$ couplings. The 95\% confidence intervals for the $\mathrm{CP}$ conserving couplings $h_{3}^{\gamma}, h_{4}^{\gamma}, h_{3}^{Z}$, and $h_{4}^{Z}$ are presented in Figure $6[13]$.

\section{Summary}

The diboson production cross sections are measured and are found to be consistent with the SM predictions. Data 

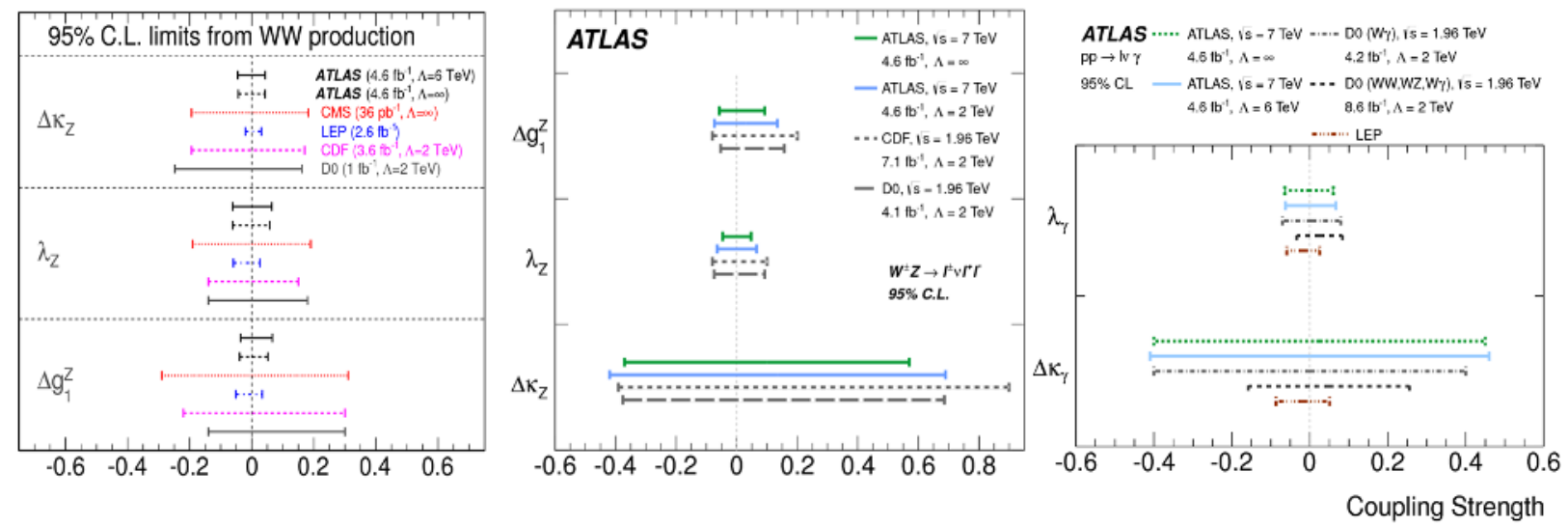

Figure 5. The 95\% CL intervals for charged aTGCs in $W W Z$ and $W W \gamma$ vertices derived from $W W$ (left) [7], $W Z$ (middle) [8], and $W \gamma$ (right) [13] channels.
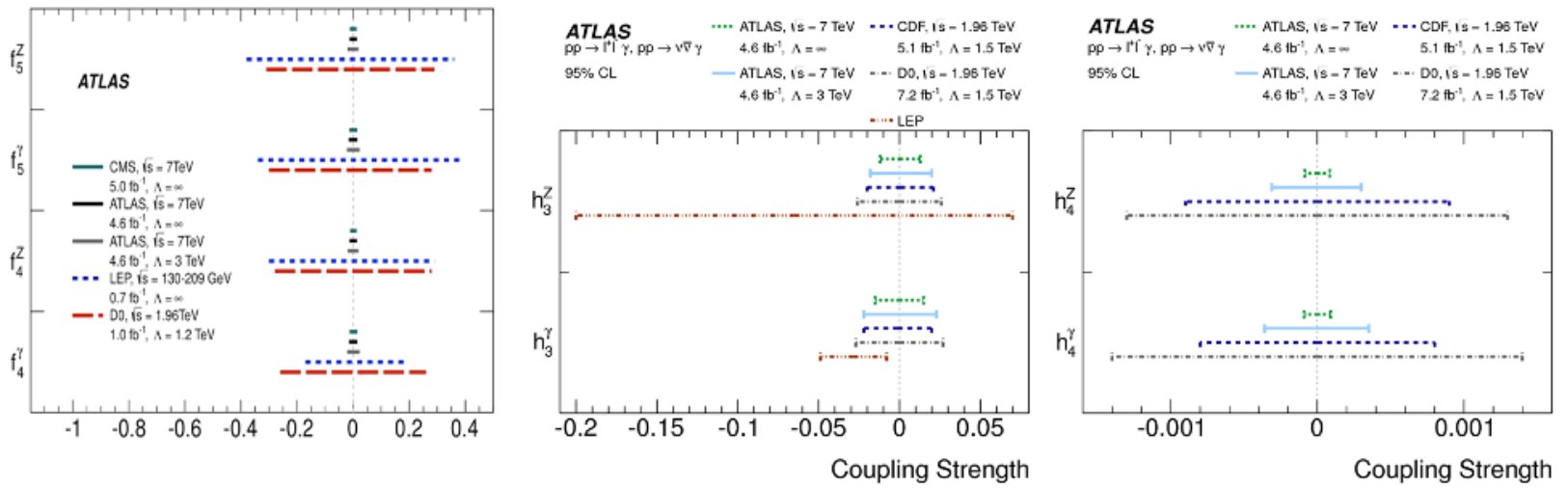

Figure 6. The 95\% CL intervals for neutral aTGCs derived from data of $Z Z$ (left) [11] and $Z \gamma$ (middle, right) [13] channels.

agree with SM predicted triple gauge couplings and $95 \%$ C.L. limits are set for the anomalous couplings.

\section{References}

[1] ATLAS Collaboration, JINST 3, S08003 (2008).

[2] J. Ellison et al. Ann. Rev. Nucl. Part. Sci. 4833 (1998).

[3] M.S. Neubauer, Annu. Rev. Nucl. Part.Sci. 61, 223 (2011), and references there in.

[4] J.M. Campbell el. al, JHEP 07018 (2011).

[5] H.-L. Lai et al., Phys. Rev. D 82074024 (2010).

[6] A. Martin et al., Eur. Phys. J. C 63189 (2009).
[7] ATLAS Collaboration, Phys. Rev. D 87112001 (2013).

[8] ATLAS Collaboration, Eur. Phys. J. C 72:2173 (2012).

[9] ATLAS Collaboration, ATLAS-CONF-2013-021.

[10] ATLAS Collaboration, ATLAS-CONF-2012-157.

[11] ATLAS Collaboration, JHEP 03128 (2013).

[12] ATLAS Collaboration, ATLAS-CONF-2013-020.

[13] ATLAS Collaboration, Phys. Rev. D 87112003 (2013).

[14] K. Hagiwara et al., Nucl. Phys. B282 253 (1987).

[15] U. Baur, D. Rainwater, Phys. Rev. D 62:113011 (2000).

[16] U. Baur, E.L. Berger, Phys. Rev. D 47:4889 (1993).

[17] G. Gounaris et al., arXiv:hep-ph/9601233. 